PROKLA-Redaktion

\title{
Editorial: Osteuropa und der Westen
}

Viele, vor allem westliche Einschätzungen der osteuropäischen Reformpolitik waren noch bis vor kurzem von der selbstgewissen Ansicht geprägt, daß die postkommunistische Transformation in den fortgeschritteneren Reformstaaten im wesentlichen abgeschlossen sei. Die entscheidende Aufgabe der Politik wurde im Bruch mit den zentralen Institutionen des Sozialismus gesehen - und die Kommunistischen Parteien und die Planbürokratien waren auch innerhalb kürzester Zeit von der Bildfläche verschwunden. Die konstruktiven Leistungen des Staats sollten sich im Großen und Ganzen darauf beschränken, die Basisinstitutionen eines »demokratischen Kapitalismus « zu etablieren. Die Fortschritte einzelner Länder wurden an einem Entwicklungspfad mit klar festgelegten Wegmarken gemessen, dessen Richtung alternativlos vorgezeichnet war. Nahezu alle osteuropäischen Regierungen haben sich in den Jahren nach 1989 auf einen Liberalisierungskurs verpflichtet - sei es aus eigener Überzeugung, sei es unter dem Druck der westlichen Gläubiger.

Die neoliberalen Reformprogramme gewannen ihre Überzeugungskraft angesichts der desaströsen Auswirkungen blockierter Reformen in der Ukraine und Weißrußland und nicht zuletzt durch die Hoffnung, bei konsequenten Reformen an den westeuropäischen Wohlstand an- schließen zu können. Das in den Ländern der Dritten Welt angewandte Konzept der »strukturellen Anpassung « wurde zur vorherrschenden Transformationstheorie, in der freilich entgegen dem akademischen Ritus kaum zwischen positiver Analyse und normativen Politikempfehlungen unterschieden wurde. Aus der Sicht dieses Konzepts war der Niedergang der sozialistischen Länder nur das augenfälligste Beispiel für das notwendige Scheitern staatszentrierter, von Weltmarkteinflüssen abgeschirmter Entwicklungstrategien, die zuvor bereits in Südeuropa und Lateinamerika verabschiedet worden waren. Von daher konnte das Ende des osteuropäischen Sozialismus auch als Vollendung der in den 80er Jahren eingeleiteten »neoliberalen Revolution« aufgefaßt und für die politischen Auseinandersetzungen innerhalb der westlichen Länder instrumentalisiert werden: als Argument gegen die sklerotischen Gefahren, die von Wohlfahrtsstaat, steuerlicher Umverteilung und industriepolitischen Ambitionen ausgehen. Insofern schienen die Kriterien für den Erfolg der postkommunistischen Transformationen auf der Hand zu liegen. Liberalisierung, Stabilisierung und Privatisierung - so lautete die trinitarische Formel, die den empirischen Analysen der internationalen Finanzorganisationen zugrunde gelegt wurde. Die optimale Se- 
quenz der radikalen Reformschritte sollte von der Liberalisierung der Preise und der Öffnung des Außenhandels über die Stabilisierung des Preisniveaus und der öffentlichen Ausgaben zur Ausweitung des privaten Sektors führen. Konsequenter Reformpolitik wurde in Aussicht gestellt, innerhalb von zwei Jahren die Inflation unter Kontrolle zu bringen und nach drei Jahren einen neuen Wachstumstrend zu begründen, der zugleich die Tragfähigkeit der neuen demokratischen Institutionen erhöhen würde. Vor diesem Hintergrund wurden der Tschechischen Republik, Polen und Slowenien die größten Fortschritte in Richtung Marktwirtschaft und Demokratie bescheinigt. Aber auch das gradualistische Ungarn und die Slowakische Republik schienen weit fortgeschritten zu sein. Und selbst Rußland wurde von westlichen Experten, welche die russische Regierung von 1991 bis 1994 beraten hatten, ein erfolgreicher Übergang zur Marktwirtschaft attestiert. Aus heutiger Sicht wirkt diese Einschätzung bestenfalls naiv. Der russische Staat hat die Souveränität über weite Bereiche der Innenpolitik verloren und seine haushaltspolitische Hohlheit an den Internationalen Währungsfonds abgetreten. Die Privatisierungspolitik hat alles andere als eine bürgerliche Klasse hervorgebracht, deren Interessen auf eine Konsolidierung rechtsstaatlicher Verhältnisse zielen. Hier, aber auch in den anderen Reformländern, hat sich die Privatisierung als Haupteinfallstor für Korruption und als das Medium erwiesen, über das alte Machtgruppen weiterhin auf ökonomische Ressourcen und politische Entscheidungen zugreifen. Um den illusorischen Gehalt des makroökonomischen Empirismus von Weltbank und IWF aufzuzeigen, braucht man nicht auf die positive Einschätzung Albaniens noch im Weltent- wicklungsbericht von 1996 zu verweisen - kurz bevor das Land in anarchischen Bandenkämpfen versank. Im jüngsten OECD-Bericht über die Tschechische Republik ist der einstige Musterschüler radikaler Reformpolitik kaum wiederzuerkennen. Die Inflationsrate ist auf zweistellige Werte hochgeschnellt, das geschätzte Wachstum wird in den nächsten Jahren unter dem der Bundesrepublik liegen. Die formelle Aufnahme in die »OECD-Welt« erweist sich als Illusion, und selbst der immer wieder zugesagte Beitritt in die Europäische Union wird in Frage gestellt.

Sicherlich reflektieren sich in den empirisch verfügbaren Daten über die Situation in den osteuropäischen Ländern kaum vermeidbare Anpassungsprobleme. Entscheidend waren jedoch die konzeptionellen Defizite der neoliberalen Reformprogramme: die Stilisierung von Märkten $\mathrm{zu}$ einem universalen Organisationsmodell und ein verkürztes Verständnis von »politischer Ökonomie«, das den Markt als Instrument gegen gesellschaftliche Ansprüche, an der Ausgestaltung der Reformpolitik mitzuwirken, einsetzt. Privatisierungen und Marktzwänge sollten die rent seeking-Koalition zwischen den Managern der alten Betriebe und den wohlfahrtspaternalistisch akkommodierten Belegschaften notfalls auch im Stil eines »aufgeklärten Autoritarismus « aufbrechen. Märkte werden zur Verkörperung des Allgemeinwohls stilisiert, dem wohlwollende Reformer gegen Sonderinteressen und populistische Versuchungen zum Durchbruch verhelfen.

In der Praxis der Reformpolitik hat sich die Illusion universaler Marktlösungen freilich zu einem Teil des Problems verkehrt, indem sie zur programmatischen Desorganisation der politischen Strukturen beigetragen haben. Rent seeking, a- 
symmetrische Informationen und Machtkämpfe, wie man nun feststellt, sind integraler Bestandteil von Märkten, deren Funktionsweise alles andere als einfach ist (vgl. dazu Bowles/Gintis in Prokla 81, 1990 und deVroey in Prokla 82, 1991). Insofern ist es nur konsequent, wenn Joseph Stiglitz, der derzeitige leitende Ökonom der Weltbank, die gegen die Realität gewandte Abstraktion selbstorganisierter Märkte als mitschuldig an dem desaströsen Verlauf der osteuropäischen Reformen ansieht (Whither Socialism?, Cambridge, Mass, 1995).

Unter dem Eindruck der postkommunistischen Krisen haben sich daher auch die Perspektiven in der Transformationsforschung verschoben. Statt von einem Abschluß der Transformation wäre eher vom Ende einer ökonomistisch reduzierten Transformationstheorie zu reden. Denn von zentraler Bedeutung erscheinen heute die außerökonomischen Voraussetzungen ökonomischer Reformen, die einerseits auf das sozialstrukturelle und politische Erbe der sozialistischen Vorgeschichte verweisen, andererseits auf komplexe Überlagerungen von kulturellen Ressourcen und zivilgesellschaftlichen Kompetenzen mit kapitalistischen Modernisierungszwängen. Melanie Tatur und Jadwiga Staniszkis verwenden das Konzept des »politischen Kapitalismus«, das bereits Max Weber für die spezifische Form der peripheren Kapitalisierung Rußlands um die Jahrhundertwende geprägt hatte, für eine vergleichende Analyse der unterschiedlichen postsozialistischen Reformpfade, die neben der Chance zur demokratischen Konsolidierung auch die Möglichkeiten der Peripherisierung, der Segmentierung der Staatsgewalt und der anarchischen Desintegration enthalten. Im postsozialistischen Kontext ist damit der Stellenwert des Staats für die Etablierung von Eigentum gemeint, die sich freilich nicht auf die juristische Sphäre im engeren Sinn, sondern auf die rechtliche Verankerung ökonomischer Macht und die Institutionalisierung von wirtschaftlichen Anreizen und Sanktionen bezieht. In dieser Hinsicht setzt sich Tatur von David Stark ab: nicht die faktische Verfügung über Eigentum und deren evolutionäre Rekombination (siehe dazu Starks Artikel in Prokla 94, 1994), sondern die Kodifizierung von Eigentumsrechten sei die kritische Schwelle der Transformation. Darum kann es in der postsozialistischen Politik auch nicht um den schlichten Abbau des Staats gehen, sondern um die Neudefinition seines Tätigkeitsbereichs. Die nicht selten zur Keimzelle eines dynamischen Privatsektors stilisierte Schattenökonomie und die informellen Organisationsformen unter dem Sozialismus sind allerdings nicht als automatische Aktivposten zu verbuchen. Der in diesem Bereich gepflegte Klientelismus kann sich vielmehr als eine schwere Belastung der neuen Institutionen erweisen, da die an Insider-Verhältnissen ausgebildete Moral der wechselseitigen Vorteilsnahme einer universalistischen Orientierung der Politik entgegen steht.

Jadwiga Staniszkis arbeitet die hohe Kontinuität der sozialistischen und postsozialistischen Politik am Beispiel Polens heraus - jenes Landes, in dem die »Schocktheraphie« erfunden wurde und auf dem nach der tschechischen Enttäuschung alle Hoffnungen ruhen. Die Auflösung der Planwirtschaft seit den frühen 80er Jahren, die Staniszkis rekonstriert, zeigt allerdings alles andere als einen klaren Schnitt zur Vergangenheit - nämlich eine in den frühen 80er Jahren einsetzende phasenweise Überführung staatlichen Eigentums in die Hände mitein- 
ander konkurrierender Interessengruppen im Schnittfeld zwischen Wirtschaft und Politik (vgl. dazu auch die frühen Analysen von Bugaj/Kowalik in Prokla 82, 1991 sowie Tittenbrun in Prokla 89, 1992). Statt von kreativen Unternehmern wird das Bild durch organisierte Märkte mit regulierten Eintrittsbedingungen und hybriden Eigentumsformen geprägt. In der gegenwärtigen Phase des politischen Kapitalismus konkurrieren dabei große politisierte und westwärts gewandte Gruppen mit dem kleinen und mittleren Kapital, das den Nährboden für einen peronistischen Populismus mit wirtschaftsnationalistischen Zügen abgeben könnte - insbesondere dann, wenn der Anpassungsdruck an die EU-Standards im Zuge der Beitrittsverfahren voll zu greifen beginnt.

Letzteres macht auch deutlich, daß sich der Transformationsprozeß in Osteuropa längst nicht mehr isoliert betrachten läßt. Nicht nur sind die politischen und wirtschaftlichen Beziehungen zu Westeuropa für die osteuropäischen Länder bei weitem am wichtigsten. Mit den angestrebten EU-Beitritten wird Westeuropa nachgerade zum Ziel der meisten osteuropäischen Länder. Grundlegende Veränderungen im Westen, wie sie mit der Einführung des Euro in den nächsten Jahren anstehen, definieren damit auch die Bedingungen der Transformation im Osten in neuer Weise. Zur besseren Konturierung der anstehenden Währungsunion zieht Elmar Altvater in seinem Beitrag einen Vergleich zur (west)deutschen Währungsreform des Jahres 1948 und zur deutschen Währungsunion von 1990.

Die kritische Funktion der Europäischen Union für den Erfolg der osteuropäischen Reformen wird von Peter Gowan hervorgehoben, wobei die wohlorganisierte Politik westeuropäischer Interessengrup- pen freilich längst die Oberhand über die großzügigen Gesten der Zeit nach 1989 gewonnen haben. Der westliche Protektionismus hat allen osteuropäischen Ländern defizitäre Handelsbilanzen beschert, und zwar auch in den Bereichen, in denen sie komparative Vorteile geltend machen könnten. Wann ein EU-Beitritt der osteuropäischen Länder möglich ist, wird nicht allein von den Bewerbern abhängen, sondern auch davon, wann die EU ihre eigenen institutionellen Reformen, vor allem im Bereich der Agrarpolitik, bewältigen wird. Welche Auswirkungen die EU-Agarpolitik auf die Transformationsprozesse in der ostdeutschen Landwirtschaft bereits hatte, wird in dem Beitrag von Katrin Küster untersucht, die dabei kritisch an den Aufsatz von Ralf Clasen (PROKLA 108, 1997) anknüpft.

Ebenfalls um eine spezifische West-Ost Beziehung geht es in dem Artikel von Katharina Müller, die die Umgestaltung osteuropäischer Rentensysteme analysiert, die weitgehend unter dem Einfluß der internationalen Finanzinstitutionen vor sich geht und lateinamerikanischen Vorbildern folgt. Dabei erhalten die osteuropäischen Gesellschaften ein Alterssicherungssystem, das zugleich darauf zielt - wie auch Peter Gowan in anderem Kontext hervorhebt - die spezifisch europäischen Sozialstaatstraditionen insgesamt auszuhebeln. Von der zukünftigen Ausgestaltung der Europäischen Union wird also nicht nur der weitere Verlauf der osteuropäischen Reformen abhängen, sondern auch die Art und Weise ihrer Rückwirkungen auf den Westen und damit auch die Fähigkeit der westeuropäischen Sozialstaaten, sich gegenüber einem deregulierten globalen Kapitalismus zu behaupten. 